\title{
Cloning, Expression and Purification of a Novel Fusion Protein Composed of Flagellin and NS5B of Hepatitis C Virus in Escherichia coli
}

\section{Host}

\author{
Amir Atapour ${ }^{1}$, Zohreh Mostafavi-Pour $^{2}$, Pooneh Mokarram ${ }^{2,3,{ }^{*}}$ and Shiva Mohammadi ${ }^{1}$ \\ ${ }^{1}$ Department of Medical Biotechnology, School of Advanced Medical Science and Technologies, Shiraz University of Medical Sciences, Shiraz, Iran \\ ${ }^{2}$ Recombinant Proteins Lab, Biochemistry Department, Faculty of Medical Biotechnology, School of Advanced Medical Science and Technologies, Shiraz University of Medical \\ Sciences, Shiraz, Iran \\ ${ }^{3}$ Colorectal Research Center, Gastroenterohepatology Research Center, Nemazi Hospital, Shiraz, Iran \\ "Corresponding author: Biochemistry Department, Faculty of Medical Biotechnology, Shiraz University of Medical Sciences, Zand St., Shiraz, Iran. Tel: +98-7132303320, Fax: \\ +98-7132303029, Email: mokaram2@gmail.com
}

Received 2018 May 29; Revised 2018 September 07; Accepted 2018 September 18.

\begin{abstract}
Background: At present, there is an increased demand for recombinant proteins and the process development for production has high potential to speed up. Then, scale up of the developed process are critical if commercial production is the desired.

Objectives: In this survey, we intend to produce a recombinant fusion protein of flagellin (fliC) and the N terminal fragment(NT300) of NS5B gene.

Methods: After expressing the fusion protein in the proper host (E. coli), sodium dodecyl sulfate-polyacrylamide gel electrophoresis (SDS-PAGE) and Western Blot were used for evaluating the expressed protein.

Results: Results showed that fliC-NT300 recombinant fusion protein was properly cloned in pET28a vector following by successfully expressed in E. coli. SDS-PAGE and Western Blotting techniques confirmed a protein with a molecular weight of $86 \mathrm{kDa}$.

Conclusions: Our data indicated that the novel recombinant fusion protein (fliC-NT300) was well produced by the pET28a vector in E. coli system.
\end{abstract}

Keywords: Recombinant Proteins, Flagellin, NS5B, Western Blotting

\section{Background}

The recombinant proteins can possess various usages including: (1) Immunization, (2) biochemical studies, (3) three-dimensional structure analysis of the protein, and (4) biotechnological and therapeutic applications; therefore, the production of recombinant proteins, as an accepted standard method is very important. Among the priorities of applied molecular researches, the production and study of the characteristics of target protein products are of great importance in the post-genomic era $(1,2)$. In this context, the use of DNA cloning technology, along with fusion tags, is applied as a common method for the expression and purification of interest recombinant proteins. One of the important achievements in biotechnology is the use of prokaryotic systems for the production of recombinant proteins. Due to numerous problems associated with the production of proteins including the use of large amounts of animal or plant tissues, low-amounts of protein products, as well as the difficulty of the purifi- cation, DNA recombinant technology has been allowed to solve many of these issues $(3,4)$. Therefore, after the production and purification of large quantities of interest protein, it is possible to determine some of characteristics such as physico-chemical properties and its use in industrial processes as well as the trend of commercial development $(1,5)$. Although there are several expression systems such as eukaryotic and prokaryotic for the production of recombinant proteins, one of the suitable and most widely used hosts is E. coli. On the other hand, the pET system is a significant and most common expression vector applied to facilitate the recombinant protein production (5-8). In this study, two different molecules were used to produce recombinant protein: including non-structural protein $5 \mathrm{~B}$ (NS5B) of HCV an RNA dependent RNA polymerase (RdRp), which is responsible for the replication of the hepatitis $C$ virus genome and flagellin type $\mathrm{B}$ (fliC) from Pseudomonas aeruginosa (9-13). Therefore, in this paper, we attempted to design an efficient construct to produce high yield and pu- 
rified of a novel HIS-Tag recombinant fusion protein (fliCNT300) in E. coli host (BL21).

\section{Methods}

\subsection{Construction of Recombinant Fusion Gene}

PCR amplification of the NT300 fragment was first done by a set of primers designed as follows: NT300 F: 5-GACGAATTCGGTACCAAGCTTTCAATGTC-3 and NT300 R: 5TTTCTCGAGGCGGGCTTTAATATAACACGTCAGG-3.

The pET28a expression vector (Biomatik, Ontario, Canada), containing the full coding sequence of NS5B gene from hepatitis $\mathrm{C}$ virus (HCV), was used as a template. The EcoR1 restriction site in forward primer and Xho1 restriction sites in reverse primer were underlined. PCR reaction carried out under the following program: $94^{\circ} \mathrm{C}$, $30 \mathrm{~s} ; 61^{\circ} \mathrm{C}, 30 \mathrm{~s} ; 72^{\circ} \mathrm{C}, 2$ min for a total of 35 cycles. After purification of PCR product of NT300 using gel extraction kit (Fermentas, Thermo Fisher Scientific, USA), the product was cloned into the EcoR1/Xho1 sites of expression vector pET28a (+). The pET28a vector (QIAGEN, Germany) contained full coding sequence of fliC as a template. Primers designed for reaction were as follows:

fliC F: 5-CCTGGATCCGAAGATGACGTTGAGTGGA-3

fliC R: 5-TGCGGTACCTTTGTAGAAGGCTTTGTATTC-3

The BamH1 and Kpn1 restriction sites were included in forward and reverse primers, respectively. PCR program for amplification including: for $30 \mathrm{~s}$ at $94^{\circ} \mathrm{C}$; $35 \mathrm{~s}$ at $62^{\circ} \mathrm{C}$; $1: 45 \mathrm{~min}$ at $72^{\circ} \mathrm{C}$, for a total of 35 cycles. The presence of the amplified product on gel electrophoresis was confirmed and then, the fliC fragment cut and purified using the gel extraction kit (Fermentas, Thermo Fisher Scientific, USA). To generate pET28a (fliC-NT300), the fliC fragment was digested with BamH1 and Kpn1 and then, cloned into the BamH1/Kpn1 sites of expression vector pET28a-NT300, upstream of NT300 fragment. After that, ligation product transformed into E. coli DH5a competent cells followed by PCR and restriction analysis with Nco1 and Xho1 enzymes in order to screen. The sequence integrity of positive clones were checked by DNA sequence analysis (Bioneer, Korea).

\subsection{Expression and Purification of the Recombinant fliC-NT300 Protein}

After transforming pET28a-fliC-NT300 fusion plasmid into E. coli BL21(DE3), a single transformed colony of recombinant E. coli (DE3) cells was inoculated into Luria Broth (LB) media containing $100 \mu \mathrm{g} / \mathrm{mL}$ kanamycin (Sigma, Germany) overnight. When the optical density (OD at $600 \mathrm{~nm}$ ) of the culture reached $0.6(\mathrm{OD}=0.6)$, the expression of recombinant protein was induced by addition of isopropylD-thiogalacto-pyranoside (IPTG) (Fermentas, USA) to a final concentration of $1 \mathrm{mM}$ and incubation continued at $37^{\circ} \mathrm{C}$ for $4 \mathrm{~h}$. Bacterial cells were collected by centrifugation (10000 $\mathrm{g}$ for $5 \mathrm{~min}$ ) and the cell pellets were further subjected to sodium dodecyl sulfate polyacrylamide gel electrophoresis (SDS-PAGE) to analyze the expression of the recombinant proteins.

\subsection{SDS-PAGE and Western Blot Analysis}

SDS-PAGE was used to analyze the proper expression of fliC-NT300 fusion protein by the method as previously instructed (11). Briefly, $20 \mu \mathrm{L}$ of the purified fractions were suspended in $20 \mu \mathrm{L}$ of loading buffer, heated for 5 min at $100^{\circ} \mathrm{C}$. Then, $20 \mu \mathrm{L}$ of each sample was used for loading on $13 \%$ SDS-PAGE gel. Western Blot analysis, using horseradish peroxidase (HRP), conjugated anti-polyhis antibody (Sigma, USA), with an appropriate (1:3000) dilution, was performed as described previously (10), with some modifications. The Horseradish Peroxidase color development reagent, DAB (3,3'-diaminobenzidine) solution (0.05\%) with $30 \mu \mathrm{L}$ hydrogen peroxidase (30\%) as an enzyme substrate was used for detecting bounds to nitrocellulose membrane. This substrate develops a brown insoluble product on the membrane surface after exposure to horseradish peroxidase conjugated antibodies.

\section{Results}

\subsection{Cloning of rfliC-NT300 in a Prokaryotic Expression Vector}

To produce rfliC-NT300 fusion gene, the N-terminal of NS5B (NT300) with 900 bp size was amplified by PCR reaction and inserted into the restriction sites (EcoRland Xho1) of vector pET28a. Then, plasmid contain N-terminal of NT300 was purified by plasmid extraction kit and electrophoresis of PCR products showed the length of approximately $900 \mathrm{bp}$ for NT-300 fragment. In addition, the same experiments were done for flic fragment and the band, about 1470 bp on electrophoresis gel, was detected. This fragment cloned into the BamH1/Kpn1sites of expression vector pET28a-NT300, upstream of NT300 fragment. As a result, we generated a fusion protein consisting of the fliC gene linked to the N-terminal end of NS5B gene (NT300) using the restriction enzymes method. Our construct consists of fliC- NT-300 fragments and poly-his tag (Figure 1). Final PCR product with band size 2720 bp (Figure 2) and double digestion by the restriction enzymes (BamH1/Xho1) with band near 2365 bp were shown in Figure 3. 
$\mathrm{N}$

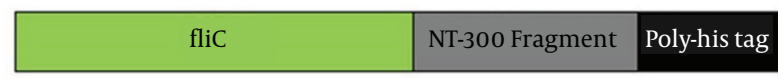

Figure 1. Recombinant fusion construct; schematic diagram of recombinant fusion construct consist of flagellin (fliC), NT300 fragment and poly-his tag

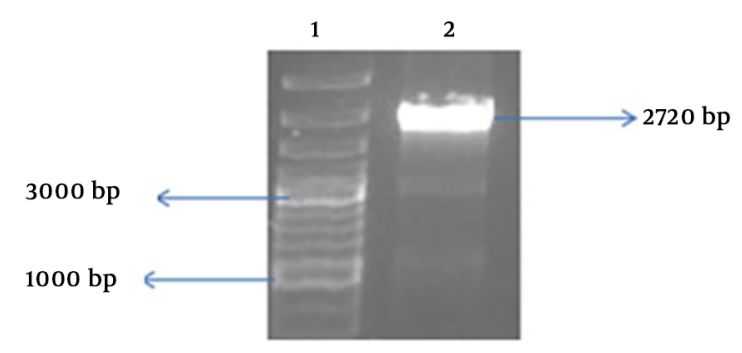

Figure 2. 1, PCR product of recombinant fusion gene; DNA ladder $1 \mathrm{~Kb} ; 2$, $\mathrm{PCR}$ product of fusion fliC-NT300 (2720 bp)

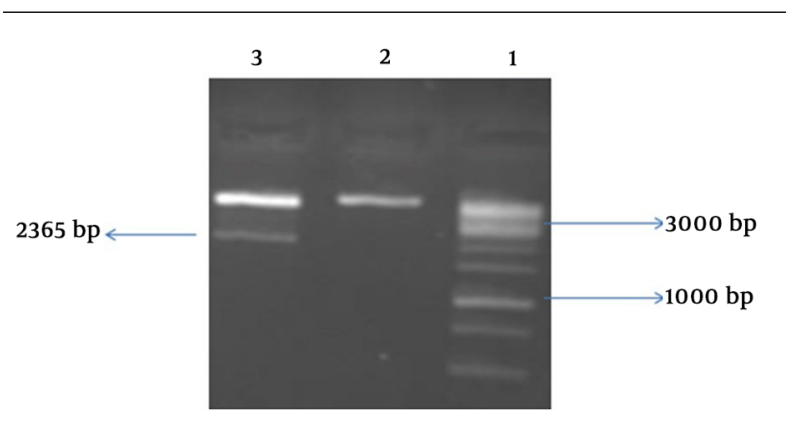

Figure 3. Double digestion analysis; 1 , DNA ladder $1 \mathrm{~Kb}$; 2 , uncut vector; 3 , vector digested by (BamH1/Xho1) enzymes (2365 bp band)

\subsection{Expression and Purification of the Recombinant rfliC-NT300 Protein}

The correct sequence of the recombinant gene was confirmed by DNA sequencing (Bioneer, Korea), then, the fusion protein fliC-NT300 was expressed in E. coli BL21 (DE3) strain and purified. For this reason the cells containing recombinant plasmid were cultured in LB medium containing $100 \mu \mathrm{g} / \mathrm{mL}$ kanamycin at $37^{\circ} \mathrm{C}$ until the OD (600 $\mathrm{nm}$ ) reached 0.6 , then, induction was done using $1 \mathrm{mM} \mathrm{IPTG}$ at $37^{\circ} \mathrm{C}$ for 6 hours. Analysis of recombinant protein was done by SDS-PAGE method. The recombinant fliC-NT300 protein was run on a polyacrylamide gel electrophoresis (12.5\%) and stained by coomassie blue. This recombinant protein, with a molecular weight of $86 \mathrm{kDa}$, can be seen on the SDS-PAGE gel (Figure 4). Under denaturing conditions and using Ni-NTA affinity column, the recombinant protein his-tagged fliC-NT300 was successfully puri- fied (Figure 4). Western Blotting technique was performed by anti-His antibody to make sure the appropriate expression of fliC-NT300 recombinant protein and displayed the existence of main band with expected size ( $86 \mathrm{kDa}$ ) corresponding to this protein in the induced bacterial lysate (Figure 5). Purified samples were confirmed by Western Blot analysis (Figure 5).

\section{Discussion}

Many of biopharmaceutical protein products and industrial enzymes such as monoclonal antibodies and antibody fragments, blood clotting factors, thrombolytic agents, vaccines, hormones, interferons, interleukinbased products, growth factors, nucleic-acid based products, and therapeutic enzymes are recombinant proteins (14-16). Appropriate and efficient strategies for the production of high amounts and high-quality of recombinant proteins are important. Several factors affect the expression of recombinant protein, one of the most important factors that can increase the concentration of target protein is selection of the appropriate expression host. From conventional expression systems, prokaryotic, yeast, insects, and mammals, the E. coli expression system is one of a well-established system. The main causes for

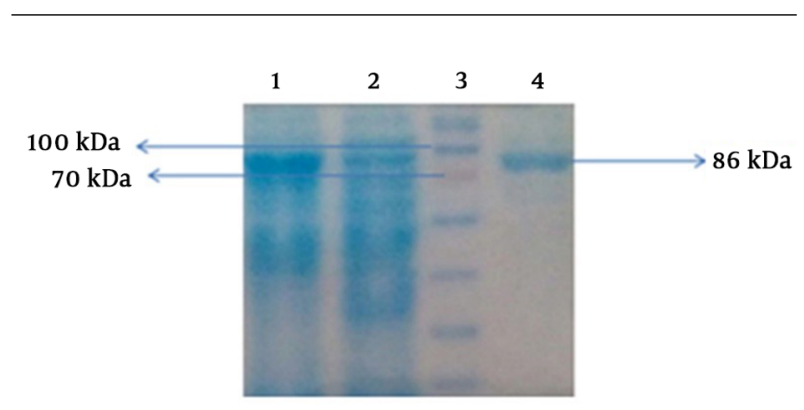

Figure 4. SDS-PAGE analysis; 1 , induce sample; 2 , non-induced sample; 3 , protein ladder; 4, purified protein

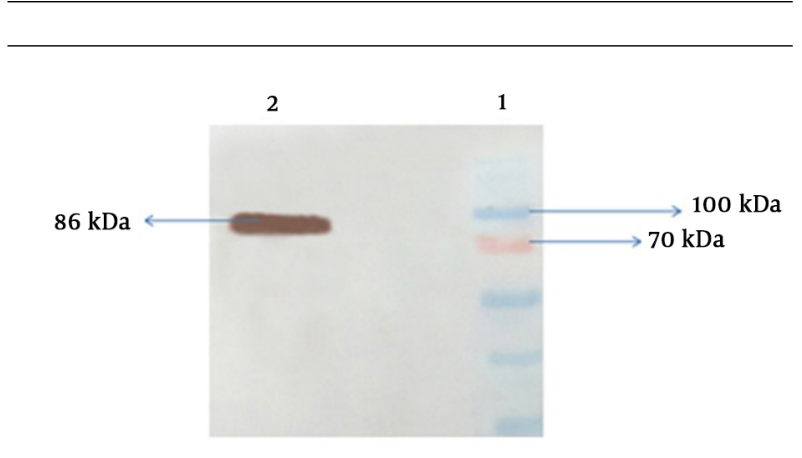

Figure 5. 1, Western Blot analysis, protein ladder; 2, BL21 without plasmid 
selection of the E. coli expression system is due to the ability to grow rapidly, low cost culture conditions, easy genetic manipulation, high expression levels of target gene, accessibility of versatile plasmid vector, a wellknowledge of biochemical, and genetic characterizations $(2,5,6)$. A variety of cloning and expression vectors are available for production of recombinant proteins. One of the most powerful and very popular systems that is widely used for cloning and expression of recombinant proteins in E. coli is the pET system. The pET system utilizes a strong promoter (bacteriophage T7 promoter), in which the target gene is cloned behind the T7 promoter and expression induced by the T7 polymerase RNA in the host source. In successful cases and a few hours after induction, the desired protein can represent $50 \%$ of the total cell protein. Another important benefit of this system is that in the non-induce state, the transcription of the desired gene becomes silent $(1,3,4)$. The E. coli expression system has common features with other vectors, including an origin of replication, promoter, a selection marker (e.g. antibiotic resistance gene), translation initiation site, 50 untranslated region (50UTR), and N-terminal codons. Unlike other elements mentioned above, fusion tags in the expression vectors can be expressed along with the target gene, which it can along with other factors; promoters, 50UTR, N-terminal codons have a significant effect on transcription, protein yields, solubility, and purification. Different tags are used to facilitate the expression and purification of recombinant proteins in host Escherichia coli, including Thioredoxin (Trx), 6xHIS-Tag, small ubiquitinlike modifier (SUMO), glutathione S-transferase (GST), green fluorescent protein (GFP), HaloTag, and maltose binding protein (MBP). Among these, due to some of the properties such as commercially available expression vectors and their downstream processing systems and more than $80 \%$ purity can be captured in a one chromatographic phase, the 6xHIS-Tag as one of the most popular tags is used (3-5). Different approaches can be used for cloning of the heterologous gene in suitable vector such as restriction enzyme (RE)-based cloning, recombination-based cloning, and annealing-based or ligation-independent cloning (LIC). Researchers prefer to use the restriction enzyme (RE)-based cloning technique compared to traditional digestion-ligation methods, due to several advantages including simple, efficient, easier to set up, universal and cost-effective. Therefore, in this study, RE-based cloning method was applied (6). After amplification of the heterologous gene using specific primers, ligation process (ligation of the heterologous gene and vector) was done by ligase enzyme and then, transformed into E. coli competent cells. Therefore, we were able to express and produce high levels of fliC-NT300 fusion protein in the pET28a system under the powerful T7 promoter. In addition, due to well-known genetics, cheap, and fast cultivation of E. coli BL21 (DE3), this system is used as a potent and versatile system $(2,3,5)$. SDS-PAGE results showed that recombinant fusion protein was considerably expressed in cell lysate to compare with non-induced samples. In addition, the presence of the C-terminal 6xHIS-Tag, in the fusion protein, could help detect fusion protein in Western Blot analysis using anti-his conjugate-antibody followed by purification of fusion protein using Ni-NTA affinity column.

\subsection{Conclusions}

Amplification, cloning, and expression of fliC-NT300 fusion protein were carried in E. coli host, successfully. In addition, it is essential that the other studies, including in vitro and in vivo, be carried out on this recombinant protein in the future.

\section{Footnotes}

Authors' Contribution: All authors contributed in drafting and revising the manuscript.

Conflict of Interests: The authors declare that they have no conflict of interest.

Funding/Support: The present article was extracted from the Ph.D. thesis written by Amir Atapour and was financially supported by Shiraz University of Medical Sciences grants number 93-7369.

\section{References}

1. Luo D, Wen C, Zhao R, Liu X, Liu X, Cui J, et al. High level expression and purification of recombinant proteins from Escherichia coli with AKTAG. PLoS One. 2016;11(5). e0156106. doi: 10.1371/journal.pone.0156106. [PubMed: 27214237]. [PubMed Central: PMC4877045].

2. Schumann W, Ferreira LCS. Production of recombinant proteins in Escherichia coli. Genet Mol Biol. 2004;27(3):442-53. doi: 10.1590/s141547572004000300022.

3. Joseph BC, Pichaimuthu S, Srimeenakshi S, Murthy M, Selvakumar $\mathrm{K}$, Ganesan M, Manjunath SR. An overview of the parameters for recombinant protein expression in Escherichia coli. J Cell Sci Therap. 2015;6(5):1. doi: 10.4172/2157-7013.1000221.

4. Young CL, Britton ZT, Robinson AS. Recombinant protein expression and purification: A comprehensive review of affinity tags and microbial applications. Biotechnol J. 2012;7(5):620-34. doi: 10.1002/biot.201100155. [PubMed: 22442034].

5. Rosano GL, Ceccarelli EA. Recombinant protein expression in Escherichia coli: Advances and challenges. Front Microbiol. 2014;5:172. doi: 10.3389/fmicb.2014.00172. [PubMed: 24860555]. [PubMed Central: PMC4029002].

6. Jia B, Jeon CO. High-throughput recombinant protein expression in Escherichia coli: Current status and future perspectives. Open Biol. 2016;6(8). doi: 10.1098/rsob.160196. [PubMed: 27581654]. [PubMed Central: PMC5008019]. 
7. Mierendorf RC, Yeager K, Novy R. The pET system: Your choice for expression. Innovations. 1994;1(1):1-3.

8. Mierendorf RC, Morris BB, Hammer B, Novy RE. Expression and purification of recombinant proteins using the pET system. Methods Mol Med. 1998;13:257-92. doi: 10.1385/0-89603-485-2:257. [PubMed: 21390849].

9. Atapour A, Mokarram P, MostafaviPour Z, Hosseini SY, Ghasemi Y, Mohammadi S, et al. Designing a fusion protein vaccine against HCV: An in silico approach. Int JPept Res Ther. 2018:1-12. doi: 10.1007/s10989-0189735-4.

10. Hajizadeh MR, Mokarram P, Kamali Sarvestani E, Bolhassani A Mostafavi Pour Z. Recombinant nonstructural 3 protein, rNS3, of hepatitis C virus along with recombinant GP96 induce IL-12, TNFalpha and alpha5integrin expression in antigen presenting cells. Hepat Mon. 2013;13(6). e8104. doi: 10.5812/hepatmon.8104. [PubMed 24032046]. [PubMed Central: PMC3768236].

11. Hemmati M, Seghatoleslam A, Rasti M, Ebadat S, Mosavari N, Habibagahi $\mathrm{M}$, et al. Expression and purification of recombinant Mycobacterium Tuberculosis (TB) antigens, ESAT-6, CFP-10 and ESAT- 6/CFP-10 and their diagnosis potential for detection of TB patients. Iran Red Crescent Med J. 2011;13(8):556-63. [PubMed: 22737527]. [PubMed Central: PMC3372000].
12. Atapour A, Mokarram P, Mostafavi-Pour Z, Ramezani A. Molecular cloning, expression, and purification of a recombinant fusion protein (rNT-gp96-NT300). Bio Pharm Int. 2017;30(10):38-44.

13. Savardashtaki A, Sharifi Z, Hamzehlou S, Farajollahi MM. Analysis of immumoreactivity of heterologously expressed non-structural protein 4B (NS4B) from hepatitis C virus (HCV) genotype 1a. Iran J Biotechnol. 2015;13(4):32-7. doi: 10.15171/ijb.1321. [PubMed: 28959307]. [PubMed Central: PMC5458682].

14. Ferrer-Miralles N, Villaverde A. Bacterial cell factories for recombinant protein production; expanding the catalogue. Microb Cell Fact. 2013;12:113. doi:10.1186/1475-2859-12-113. [PubMed: 24245806]. [PubMed Central: PMC3842683].

15. Jozala AF, Geraldes DC, Tundisi LL, Feitosa VA, Breyer CA, Cardoso SL, et al. Biopharmaceuticals from microorganisms: From production to purification. Braz I Microbiol. 2016;47 Suppl 1:51-63. doi: 10.1016/j.bjm.2016.10.007. [PubMed: 27838289]. [PubMed Central: PMC5156500]

16. Sharma SS, Blattner FR, Harcum SW. Recombinant protein production in an Escherichia coli reduced genome strain. Metab Eng. 2007;9(2):133-41. doi: 10.1016/j.ymben.2006.10.002. [PubMed: 17126054]. [PubMed Central: PMC3710453]. 